\title{
Higher Recurrence Rate of Acute Coronary Syndrome in Patients with Multiple-Time Myocardial Infarction
}

\author{
Shigeto Namiuchi, ${ }^{1}$ MD, Shinichiro Sunamura, ${ }^{1}$ MD, Atsushi Tanita, ${ }^{1}$ MD, Ryoichi Ushigome, ${ }^{1}$ MD, \\ Kazuki Noda, ${ }^{1}$ MD and Toru Takii, ${ }^{1}$ MD
}

\begin{abstract}
Summary
The recurrence rate of acute coronary syndrome (ACS) in patients after first-time myocardial infarction (MI) is over ten times higher than in the general population. However, it is unclear whether patients with multiple-time MI have an even higher recurrence rate of MI. This study aimed to compare the recurrence rate in patients with multiple-time MI with the rate in patients after first-time MI. We retrospectively studied 794 consecutive MI patients who were discharged. Recurrent ACS was investigated in patients with previous MI $(n=$ $46)$ and without previous MI $(n=748)$. During the follow-up periods (mean \pm SD: $757 \pm 733$ days), recurrent ACS occurred in 47 cases without previous MI and in 7 cases with previous MI. Kaplan-Meier analysis revealed that the risk of recurrent ACS was significantly higher in patients with previous MI than in patients without previous MI. ACS recurrence rates one year from the onset were $4.2 \%$ in patients without previous MI and $11.9 \%$ in patients with previous MI. Landmark analysis revealed that the higher recurrence rate in patients with previous MI was as high as $14.1 \%$ from 1 year after the onset to 2 years. In conclusion, the risk of recurrent ACS may be significantly higher in patients with multiple-time MI than in patients after first-time MI.
\end{abstract}

(Int Heart J 2021; 62: 493-498)

Key words: Ischemic heart disease, Recurrent ACS, High-risk patients

$\mathrm{D}$ espite recent improvements in myocardial infarction (MI) treatment, ischemic heart disease (IHD) is recognized as a major public health concern in developed countries. According to World Health Organization projections, IHD will continue to be the leading cause of death (13.4\% of global death) in $2030 .{ }^{1)}$ In Japan, deaths from heart disease in 2018 comprised $15.3 \%$ of all deaths (167.6 per 100,000 population), and deaths from acute myocardial infarction (AMI) as a single disease accounted for $2.5 \%$ of the total deaths. ${ }^{2}$

Although the incidence and the mortality of MI are decreasing during the last few decades in Western countries, ${ }^{3,4)}$ the age-adjusted incidence of MI in Japan has been flat and in-hospital mortality has recently increased slightly. $\left.{ }^{5}\right)$ The Japan Public Health Center-based prospective (JPHC) study has reported that age-adjusted incidence rates of coronary heart disease (CHD) were 98.8 in men and 21.3 in women per 100,000 patients per year. ${ }^{6}$ Several other studies report on the incidence of MI in Japan. ${ }^{7-9)}$ According to these studies, approximately $0.1 \%$ of Japan's general population will develop MI in a year, although accurate assessment is difficult.

Secondary prevention is important after the onset of the first MI. However, some patients experience recurrent MI, which deteriorates cardiac function and increases the risk of death and possibility of heart failure. Moreover, approximately $1-4 \%$ of patients in Japan developed recurrent MI within one year after the first $\mathrm{MI},{ }^{10-13)}$ which is over ten times as high as the general population's incidence rate. However, it has not been reported whether patients with multiple-time MI have an even higher recurrence rate of MI. The study aimed to compare the recurrence rate in patients with multiple-time MI than in patients after first-time MI.

\section{Methods}

Study population: Altogether, 854 patients with acute MI according to the universal definition ${ }^{14)}$ were consecutively admitted to our hospital from January 2012 to December 2019. The cases with in-hospital death (60 patients) were excluded, and the remaining 794 patients were divided into two groups to compare the recurrence rate: patients with previous MI (multiple-time MI patients) and patients without previous MI (first-time MI patients). For supplementary analysis of the control of coronary risk factors, the patients were divided into four groups according to the presence or absence of previous MI and recurrent ACS. Low-density lipoprotein (LDL) cholesterol and hemoglobin A1c (HbA1c) levels in each group were examined on admission and approximately one year after the MI onset, retrospectively.

From the ${ }^{1}$ Department of Cardiology, Sendai City Medical Center, Sendai Open Hospital, Sendai, Japan.

Address for correspondence: Shigeto Namiuchi, MD, Department of Cardiology, Sendai City Medical Center, Sendai Open Hospital, 5-22-1 Tsurugaya, Miyagino-ku, Sendai, Miyagi 983-0824, Japan. E-mail: nami@openhp.or.jp

Received for publication July 31, 2020. Revised and accepted November 12, 2020.

Released in advance online on J-STAGE May 1, 2021.

doi: 10.1536/ihj.20-546

All rights reserved by the International Heart Journal Association. 
Table I. Patient Characteristics and Laboratory Findings

\begin{tabular}{|c|c|c|c|c|}
\hline & $\begin{array}{l}\text { Overall cohort } \\
\quad(n=794)\end{array}$ & $\begin{array}{l}\text { With previous MI } \\
\qquad(n=46)\end{array}$ & $\begin{array}{l}\text { Without previous MI } \\
\qquad(n=748)\end{array}$ & $P$ \\
\hline Age (years) & $67 \pm 14$ & $72 \pm 11$ & $67 \pm 14$ & 0.016 \\
\hline Male & $590(74 \%)$ & $37(80 \%)$ & $553(74 \%)$ & 0.33 \\
\hline Body mass index $\left(\mathrm{kg} / \mathrm{m}^{2}\right)$ & $24.3 \pm 3.7$ & $24.4 \pm 3.7$ & $24.2 \pm 3.7$ & 0.72 \\
\hline Dyslipidemia & $466 / 794(59 \%)$ & $34 / 46(74 \%)$ & $432 / 748(58 \%)$ & 0.031 \\
\hline Diabetes mellitus & $231 / 794(29 \%)$ & $21 / 46(46 \%)$ & $210 / 748(28 \%)$ & 0.011 \\
\hline Hypertension & $499 / 794(63 \%)$ & $37 / 46(80 \%)$ & $462 / 748(62 \%)$ & 0.011 \\
\hline Previous MI (times) & & $1.1 \pm 0.3$ & 0 & $<0.0001$ \\
\hline Previous HF admission & $9 / 794(1 \%)$ & $4 / 46(9 \%)$ & $5 / 748(1 \%)$ & $<0.0001$ \\
\hline Previous stroke & $84 / 794(11 \%)$ & $6 / 46(13 \%)$ & $78 / 748(10 \%)$ & 0.58 \\
\hline Chronic hemodialysis & $9 / 794(1 \%)$ & $1 / 46(2 \%)$ & $8 / 748(1 \%)$ & 0.49 \\
\hline Chronic AF/Paroxysmal AF & $37 / 794(5 \%) / 91 / 794(11 \%)$ & $4 / 46(9 \%) / 5 / 46(11 \%)$ & $33 / 748(4 \%) / 86 / 748(12 \%)$ & 0.41 \\
\hline Hemoglobin (g/dL) & $13.9 \pm 2.0$ & $14.0 \pm 1.9$ & $13.9 \pm 2.0$ & 0.87 \\
\hline Creatinine (mg/dL) & $0.83[0.69,1.0]$ & $0.85[0.70,1.1]$ & $0.83[0.69,1.0]$ & 0.38 \\
\hline Creatine kinase (U/L) & $136[82,290]$ & $143[75,239]$ & $135[82,292]$ & 0.49 \\
\hline BS & $164 \pm 71$ & $176 \pm 75$ & $163 \pm 71$ & 0.27 \\
\hline HbA1c $(\%)$ & $6.3 \pm 1.3$ & $6.8 \pm 1.6$ & $6.3 \pm 1.3$ & 0.017 \\
\hline Total cholesterol (mg/dL) & $199 \pm 52$ & $194 \pm 56$ & $200 \pm 52$ & 0.52 \\
\hline LDL-cholesterol (mg/dL) & $122 \pm 39$ & $121 \pm 51$ & $122 \pm 38$ & 0.91 \\
\hline HDL-cholesterol (mg/dL) & $45 \pm 12$ & $45 \pm 14$ & $45 \pm 12$ & 0.92 \\
\hline Triglyceride (mg/dL) & $111[71,176]$ & $94[65,196]$ & $112[71,174]$ & 0.60 \\
\hline BNP (pg/mL) & $47[15,161]$ & $110[24,401]$ & $46[15,152]$ & 0.066 \\
\hline
\end{tabular}

MI indicates myocardial infarction; HF, heart failure; AF, atrial fibrillation; BS, blood sugar; HbA1c, hemoglobin A1c; LDL, low density lipoprotein; HDL, high density lipoprotein; and BNP, brain natriuretic peptide.

Protocol: We analyzed various patient characteristics such as age, sex, body mass index (BMI), coronary risk factors, patient's status upon admission, and medication after hospitalization. Diagnoses of hypertension, diabetes mellitus (DM), and dyslipidemia were obtained from the patients' medical records or previous medical therapy histories.

The study outcome was recurrent acute coronary syndrome (ACS). Recurrent ACS was defined as fatal MI, non-fatal MI, and unstable angina requiring emergent hospitalization. Medical charts were retrospectively reviewed to obtain data on post-discharge outcomes, which had been assessed based on the patient's history of regular visits to the hospital after discharge and contact with the patient's family and family doctors. The investigation conformed to the principles outlined in the Declaration of Helsinki. The study was approved by Sendai City Medical Center's ethical committee (number 2020-0019), and all the patients provided written informed consent.

Statistical analysis: Continuous data are presented as mean \pm standard deviation (or as the median and interquartile range) and were compared using the Student's $t$ test. Categorical data are presented as percentages and were compared using the Chi-square test. Kaplan-Meier estimates were compared using log-rank tests to evaluate the associations between the existence of previous MI and the risk of recurrent MI. Landmark analyses were conducted from 0 years to one year and from one year to two years of follow-up. To investigate whether a high risk of recurrent ACS could persist for more than one year in patients with multiple-time MI, we set one year as a landmark point. Values of $P<0.05$ were considered statistically significant. Furthermore, we constructed the following three Cox proportional hazards regression models: an unadjusted model, an age- and sex-adjusted model, and a fully adjusted model. In the fully adjusted model, we included the previous MI history and 18 variables considered influencing factors for recurrent ACS [age, sex, coronary risk factors (dyslipidemia, DM, hypertension, current smoking), LDL cholesterol at admission (mg/dL), HbA1c at admission (\%), Killip class, left ventricular ejection fraction $(\%)$, peak creatine kinase (U/L), and prescription at discharge (aspirin or thienopyridine, angiotensin converting enzyme inhibitor (ACEI) or angiotensin II receptor blocker (ARB), $\beta$-blocker, statin, calcium blocker, nitrate, and oral anticoagulant)]. We used the JMP software (JMP version 14.2.0, SAS Institute, Cary, North Carolina, USA). The data missing more than $10 \%$ at admission were brain natriuretic peptide (BNP: $n=120,15.1 \%$ ), low-density lipoprotein cholesterol (LDL-C: $n=114$, $14.4 \%$ ), triglyceride (TG: $n=98$ ), high-density lipoprotein cholesterol (HDL-C: $n=96,12.1 \%$ ), total cholesterol (TC: $n=95,12.0 \%$ ), and glycated hemoglobin (HbA1c: $n=$ $90,11.3 \%$ ).

\section{Results}

Patient characteristics, laboratory findings, and patient status: Seven hundred and ninety-four patients were investigated retrospectively. The mean patient age was $67 \pm$ 14 years, and $74 \%$ of the patients were men. Of the patients, $5.8 \%(n=46)$ had previous MI history (one time: 40 patients, two times: 6 patients). Table I compares the baseline characteristics and laboratory findings at the admission of patients with and without previous MI. The patients with previous MI were older and had a higher proportion of admission history for heart failure. The morbidity due to dyslipidemia, DM, and hypertension was significantly higher in patients with previous MI. HbAlc of 
Table II. Patient Status upon Admission and In-Hospital Management

\begin{tabular}{lcccc}
\hline & $\begin{array}{c}\text { Overall cohort } \\
(n=794)\end{array}$ & $\begin{array}{c}\text { With previous MI } \\
(n=46)\end{array}$ & $\begin{array}{c}\text { Without previous MI } \\
(n=748)\end{array}$ & $P$ \\
\hline Time from the onset to hospital (hours) & $2.4[1.0,8.8]$ & $2.6[1.2,6.6]$ & $2.3[1.0,8.8]$ & 0.29 \\
STEMI & $471 / 794(59 \%)$ & $17 / 46(37 \%)$ & $454 / 748(61 \%)$ & 0.0037 \\
Killip class (I/II/III/IV) & $670 / 61 / 36 / 27$ & $32 / 7 / 6 / 1$ & $638 / 54 / 30 / 26$ & 0.0049 \\
Killip class III/IV & $63 / 794(8 \%)$ & $7 / 46(15 \%)$ & $56 / 748(7 \%)$ & 0.060 \\
LVEF (\%) & $58 \pm 11$ & $50 \pm 12$ & $59 \pm 11$ & $<0.0001$ \\
LVEF < 50\% & $166 / 773(21 \%)$ & $20 / 44(45 \%)$ & $144 / 729(20 \%)$ & $<0.0001$ \\
Emergent CAG & $715 / 794(90 \%)$ & $40 / 46(87 \%)$ & $675 / 748(90 \%)$ & 0.47 \\
Primary PCI within 24 hours & $537 / 794(68 \%)$ & $32 / 46(70 \%)$ & $505 / 748(68 \%)$ & 0.77 \\
IABP & $58 / 794(7 \%)$ & $5 / 46(11 \%)$ & $53 / 748(7 \%)$ & 0.34 \\
PCPS & $1 / 794(0.1 \%)$ & $0 / 46(0 \%)$ & $1 / 748(0.1 \%)$ & 0.80 \\
Peak CK (U/L) & $817[256,2352]$ & $472[200,1684]$ & $851[264,2378]$ & 0.71 \\
Medication & & & & \\
$\quad$ DAPT & $588 / 794(74 \%)$ & $36 / 46(78 \%)$ & $552 / 748(74 \%)$ & 0.50 \\
ACEI/ARB & $624 / 794(79 \%)$ & $35 / 46(76 \%)$ & $589 / 748(79 \%)$ & 0.67 \\
$\quad \beta$-blocker & $511 / 794(64 \%)$ & $30 / 46(65 \%)$ & $481 / 748(64 \%)$ & 0.90 \\
Statin & $621 / 794(78 \%)$ & $37 / 46(80 \%)$ & $584 / 748(78 \%)$ & 0.71 \\
\hline
\end{tabular}

MI indicates myocardial infarction; STEMI, ST elevated myocardial infarction; LVEF, left ventricular ejection fraction; CAG, coronary angiography; PCI, percutaneous coronary intervention; IABP, intra-aortic balloon pumping; PCPS, percutaneous cardiopulmonary support; CK, creatine kinase; DAPT, dual antiplatelet therapy; ACEI, angiotensin converting enzyme inhibitor; and ARB, angiotensin receptor blocker.

Table III. LDL-Cholesterol and HbA1c Levels on Admission and One Year After the Onset

\begin{tabular}{cccccc}
\hline Previous MI & $\begin{array}{c}(-) \\
(-)\end{array}$ & $\begin{array}{c}(-) \\
(+)\end{array}$ & $\begin{array}{c}(+) \\
(-) \\
(n=4)\end{array}$ & $\begin{array}{c}(+) \\
(+) \\
(n=7)\end{array}$ & $P$ \\
& $(n=701)$ & $(n=47)$ & & & \\
\hline LDL-cholesterol $(\mathrm{mg} / \mathrm{dL})$ & & & & & \\
on admission & $123 \pm 39$ & $113 \pm 30$ & $115 \pm 47$ & $158 \pm 59$ & 0.036 \\
& $602 / 701(86 \%)$ & $37 / 47(79 \%)$ & $35 / 39(90 \%)$ & $6 / 7(86 \%)$ & \\
one year after onset & $87 \pm 25$ & $86 \pm 22$ & $84 \pm 32$ & $137 \pm 45$ & 0.0002 \\
& $448 / 701(64 \%)$ & $29 / 47(62 \%)$ & $24 / 39(62 \%)$ & $5 / 7(71 \%)$ & \\
HbA1c (\%) & & & & \\
on admission & $6.3 \pm 1.3$ & $6.3 \pm 1.3$ & $6.7 \pm 1.6$ & $6.9 \pm 1.6$ & 0.12 \\
& $626 / 701(89 \%)$ & $38 / 47(81 \%)$ & $34 / 39(87 \%)$ & $6 / 7(86 \%)$ & \\
one year after onset & $6.2 \pm 0.8$ & $6.0 \pm 0.5$ & $6.7 \pm 1.1$ & $6.9 \pm 0.4$ & 0.0037 \\
& $412 / 701(59 \%)$ & $30 / 47(64 \%)$ & $20 / 39(51 \%)$ & $4 / 7(57 \%)$ & \\
\hline
\end{tabular}

MI indicates myocardial infarction; ACS, acute coronary syndrome; LDL, low density lipoprotein; and HbA1c, hemoglobin Alc.

patients with previous MI was higher than that of patients without MI, and BNP at admission tended to be higher. There were no significant differences in lipid levels between both groups. Table II presents the patient status at the time of admission and during in-hospital management. In patients with previous $\mathrm{MI}$, the proportion of STelevated myocardial infarction (STEMI) was lower, Killip class was higher, and the left ventricular ejection fraction (LVEF) was significantly lower. The proportions of patients with emergent coronary angiography (CAG) and percutaneous coronary intervention (PCI) within 24 hours from the onset were similar in both groups. Medication upon discharge was not different between the two groups. Table III shows the LDL cholesterol and HbA1c levels on admission and approximately one year after the onset of MI in four groups, divided by previous MI and recurrent ACS. The LDL cholesterol and HbAlc levels were examined in approximately $90 \%$ of cases at the time of admission and in approximately $60 \%$ of cases one year after the onset of MI. Blood sampling was performed $342 \pm 70$ days after the onset. The LDL cholesterol level on admission and approximately one year after the onset of MI was highest in patients with previous MI and recurrent ACS compared with the other three groups. The HbA1c level one year after in patients with previous MI and recurrent ACS was similar to that in patients with previous MI and without recurrent ACS, but it tended to be high compared with the other two groups.

Clinical outcomes: During the follow-up periods (mean \pm SD: $757 \pm 733$ days; median 464 days), ACS recurrence was observed in 54 patients $(6.8 \%)$ : 47 cases $(6.3 \%)$ in patients without previous MI and 7 cases (15.2\%) in patients with previous MI. Kaplan-Meier analysis revealed that the risk of recurrent ACS was significantly higher in patients with previous MI compared with that in patients without previous MI. In patients without previous MI, the risk of recurrent $\mathrm{ACS}$ at 0.5 years, 1 year, 1.5 years, and 2 years was $2.4 \%, 4.2 \%, 5.7 \%$, and $6.3 \%$, respectively. In contrast, in patients with previous MI, the risk was higher at $5.4 \%, 11.9 \%, 15.9 \%$, and $24.4 \%$, respectively (Figure 


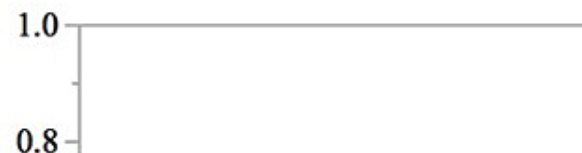

Log-rank: $P=0.012$

With previous MI, n 46

Without previous MI, n 748

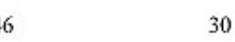

540
With previous MI

Without previous MI

Figure 1. Kaplan-Meier curves for a recurrence rate of the acute coronary syndrome (ACS) in patients with (solid line) and without (dotted line) previous myocardial infarction (MI) (Log-rank: $P=0.012$ ). The $\mathrm{X}$-axis indicates the number of patients at risk in both groups.

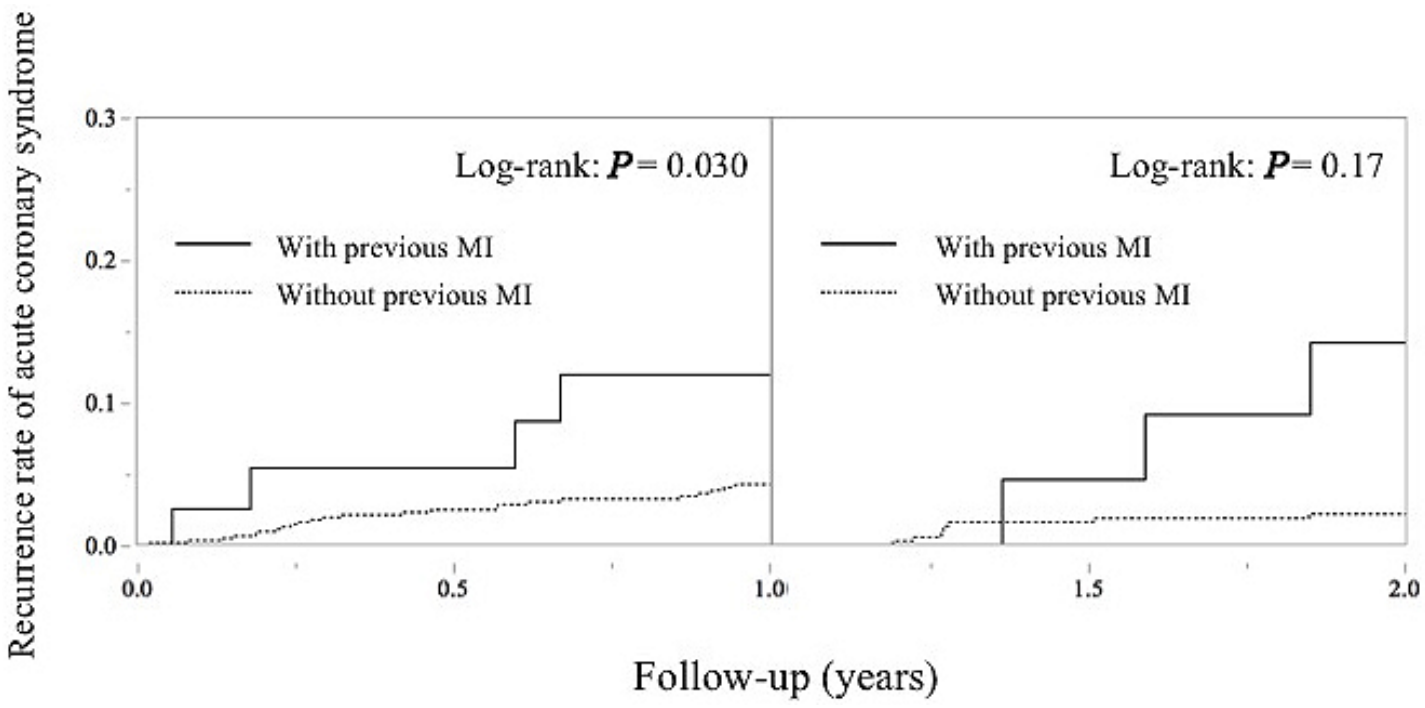

Figure 2. Landmark analyses for $0-1$ year and $1-2$ year incidence of acute coronary syndrome (ACS) recurrence.

1). Figure 2 presents landmark Kaplan-Meier analyses for 0-1 year and 1-2 year incidences of ACS recurrence. The recurrence rates in patients without previous $\mathrm{MI}$ were $1.6 \%$ from 1 year to 1.5 years and $2.1 \%$ from 1 year to 2 years, and $4.6 \%$ and $14.1 \%$ in patients with previous MI. Table IV presents the results of Cox proportional hazards models for recurrent ACS. In the unadjusted model, the hazard ratio for recurrent ACS was 2.66 [95\% confidence interval (CI) 1.20-5.89] in patients with previous MI, compared to that in patients without previous MI. The analogous hazard ratio was 2.54 (95\% CI 1.14-5.64) in the age and sex-adjusted model in patients with previous MI. Moreover, in the fully adjusted model, the risk of recurrent ACS was significantly greater in patients with previous $\mathrm{MI}$ than in patients without previous MI (hazard ratio $3.07,95 \%$ CI $1.14-8.22, P=0.026$ ). ACEI or ARB at discharge and previous MI history were significant independent variables for recurrent ACS (hazard ratio 0.40, 95\% CI $0.18-0.89, P=0.025)$. None of the coronary risk factors (dyslipidemia, DM, hypertension, and current 
Table IV. Cox Proportional-Hazard Models for Recurrent ACS (Hazard Ratio for Previous MI)

\begin{tabular}{lccc}
\hline & $\begin{array}{c}\text { Hazard } \\
\text { ratio }\end{array}$ & $95 \%$ CI & $P$ \\
\hline Unadjusted & 1.0 & & \\
Previous MI (-) & $\begin{array}{c}1.0 \\
\text { (reference) }\end{array}$ & 1.20-5.89 & 0.016 \\
Previous MI (+) & 2.66 & & \\
Age and sex-adjusted & & & \\
Previous MI (-) & 1.0 & & \\
(reference) & & & \\
Previous MI (+) & 2.54 & $1.14-5.64$ & 0.022 \\
Fully adjusted & 1.0 & & \\
Previous MI (-) & $\begin{array}{c}1.0 \\
\text { (refence) }\end{array}$ & & \\
Previous MI (+) & 3.07 & $1.14-8.22$ & 0.026 \\
\hline
\end{tabular}

ACS indicates acute coronary syndrome; MI, myocardial infarction; and CI, confidence interval. In the fully adjusted model, we adjusted for age, sex, coronary risk factors (dyslipidemia, diabetes mellitus, hypertension, current smoking), LDL cholesterol at admission (mg/dL), HbAlc at admission (\%), Killip class, LVEF (\%), peak creatine kinase (U/L), and prescription at discharge (aspirin or thienopyridine, ACE inhibitor or ARB, $\beta$-blocker, statin, calcium blocker, nitrate, and oral anticoagulant).

smoking) remained significant independent variables.

\section{Discussion}

In the most developed countries, including Japan, measures for acute MI have become important issues, and secondary prevention after first-time MI is an essential strategy. However, some patients experience recurrence of MI, which could greatly impact the patients by increasing the risk of death and/or the development of heart failure.

Information on the recurrence rate of MI is obtained from some previous studies. In Western countries, approximately $8-10 \%$ of patients reportedly developed recurrent MI within one year after the first MI. According to the Medicare database in the United States, the 1-year recurrent AMI hospitalization rate was $10.1 \%$ in patients with MI from 1999 to $2010 .{ }^{15)}$ From the data of the Danish National Registry, $8.4 \%$ of patients developed a recurrent AMI within the first year after admission for the first MI. ${ }^{16)}$

In Japan, the recurrence rate was as low as $1-4 \%$ of patients with MI. The Japanese Coronary Artery Disease Study (J-CAD study) has reported that the incidence rate of recurrent $\mathrm{MI}$ in patients who had $\mathrm{MI}$ at the time of registration was 9.4/1,000 patients/year during a 2.7-year follow-up period. ${ }^{10)}$ In the Japanese Acute Coronary Syndrome Study (JACCS), 2\% of patients with AMI had experienced re-infarction during $412 \pm 219$ days follow-up period. ${ }^{11)}$ The Osaka Acute Coronary Insufficiency Study (OACIS) has indicated that non-fatal MI had developed in $3.8 \%$ of survivors with AMI and without DM. ${ }^{12)}$ The PACIFIC registry, a prospective observational study, has reported that the incidences of fatal MI and non-fatal MI/ unstable angina were $1.6 \%$ and $5.4 \%$ during a two-year follow-up, respectively. ${ }^{13)}$

In both Japan and Western countries, the recurrence rate is higher than the incidence of first-time MI, suggesting that patients with MI are a very high-risk population for CHD. Moreover, there is a possibility that patients with multiple-time MI have a much higher recurrence rate. This study is the first investigation focused on recurrence rates in patients with multiple-time MI.

Our study revealed that patients who had experienced MI repeatedly were more likely to experience recurrence. The recurrence rate of ACS in patients with multiple-time MI was much higher than that in patients after one-time MI. In patients with multiple-time MI, the recurrence rate was $11.9 \%$ one year after MI onset and $24.4 \%$ two years after. Here, patients with previous MI had higher morbidity of coronary risk factors than patients without previous MI. Nevertheless, the lipid levels at admission were equivalent in both groups, and DM control was poorer in patients with previous MI.

Moreover, the LDL cholesterol level in patients with previous MI and recurrent ACS was significantly higher on admission and at approximately one year after the onset, although the number of cases was too low to resolve the relationship between recurrent ACS and poor control of coronary risk factors. These results may indicate that control of risk factors for secondary prevention in patients with previous MI was inadequate. Therefore, a higher-risk patient group may explain the higher recurrence rate and the insufficient control of coronary risk factors for the patient group that needed stricter control. The combination of factors on the patient side and those on the treatment side could cause a higher ACS recurrence rate. Cox proportional hazard regression models also indicated that previous MI history was a significant independent factor for recurrent MI. None of the coronary risk factors remained as significant independent variables. Previous MI history may be the result of the combined effects of coronary risk factors rather than a variable in itself. Therefore, previous MI history was a much stronger variable than each coronary risk factor; each coronary risk factor's impact may be weakly indicated when analyzed together. The previous MI history may also include other factors which have yet to be defined.

MI recurrence reportedly has been common within a year after onset. ${ }^{17,18)}$ The OACIS registry indicated that the incidence of recurrent MI per year was $2.65 \%$ for the first year and $0.91-1.42 \%$ thereafter up to 5 years. ${ }^{18)}$ Here, similar to the OACIS registry, the incidence of recurrent ACS in the first year was higher than in the second year in patients after first-time MI (the recurrence rate was $4.2 \%$ for the first year and $2.1 \%$ for the second year). However, in patients with multiple-time MI, the recurrence rate from the onset to 1 year was high (11.9\%), and the incidence rate remained high at $14.1 \%$ from one year to two years later. Hence, a high recurrence rate may persist over time in patients with multiple-time MI.

This observational retrospective study has some limitations as it was conducted at a single center. There were some missing data in this study, especially regarding laboratory findings at admission. The recurrence rate could be underestimated because this study was unable to include patients with sudden death due to MI. Further, the most important limitation is that the results were obtained from 
a relatively small number of patients. The patient population with previous MI was small, and the actual number of patients with recurrent ACS was below ten cases in two years. Additional studies with larger sample sizes are needed to validate this study's conclusions.

\section{Conclusions}

The risk of recurrent ACS may be significantly higher in patients with multiple-time MI than in patients after first-time MI.

\section{Acknowledgments}

We thank all the staff members at Sendai City Medical Center.

\section{Disclosure}

Conflicts of interest: The authors declare that there are no conflicts of interest.

\section{References}

1. Mathers CD, Loncar D. Projections of global mortality and burden of disease from 2002 to 2030. PLoS Med 2006; 3: e442.

2. Ministry of Health, Labour and Welfare. Vital Statistics (2018). Available at: https://www.mhlw.go.jp/toukei/saikin/hw/jinkou/ge ppo/nengai18/dl/gaikyou30-190626.pdf. Accessed July 31, 2020.

3. Ibanez B, James S, Agewall S, et al. 2017 ESC Guidelines for the management of acute myocardial infarction in patients presenting with ST-segment elevation: the Task Force for the management of acute myocardial infarction in patients presenting with ST-segment elevation of the European Society of Cardiology (ESC). Eur Heart J 2018; 39: 119-77.

4. Chen J, Nomand SL, Wang Y, Dyre EE, Schreiner GC, Krumholz HM. Recent declines in hospitalizations for acute myocardial infarction for Medicare fee-for-service beneficiaries: progress and continuing challenges. Circulation 2010; 121: 1322-8.

5. Cui Y, Hao K, Takahashi J, et al. Age-specific trends in the incidence and in-hospital mortality of acute myocardial infarction over 30 years in Japan. Circ J 2017; 81: 520-8.

6. Saito I, Yamagishi K, Kokubo Y, et al. Association between mortality and incidence rates of coronary heart disease and stroke: the Japan Public Health Center-based prospective (JPHC) study. Int J Cardiol 2016; 222: 281-6.

7. Hata J, Ninomiya T, Hirakawa Y, et al. Secular trends in cardiovascular disease and its risk factors in Japanese: half-century data from the Hisayama Study. Circulation 2013; 128: 1198205.

8. Rumana N, Kita Y, Turin TC, et al. Trend of increase in the incidence of acute myocardial infarction in a Japanese population: Takashima AMI Registry, 1990-2001. Am J Epidemiol 2008; 167: 1358-64.

9. Takii T, Yasuda S, Takahashi J, et al. Trends in acute myocardial infarction incidence and mortality over 30 years in Japan: report from the MIYAGI-AMI Registry Study. Circ J 2010; 74: 93100.

10. The Japanese, Coronary Artery, Disease (JCAD) Study Investigators. Current status of the background of patients with coronary artery disease in Japan. Circ J 2006; 70: 1256-62.

11. Ogawa H, Kojima S. Modern state of acute myocardial infarction in the interventional era: observational case-control studyJapanese acute coronary syndrome study (JACSS). J Cardiol 2009; 54: 1-9.

12. Nakatani D, Sakata Y, Mizuno H, et al. Impact of diabetes mellitus on rehospitalization for heart failure among survivors of acute myocardial infarction in the percutaneous coronary intervention era. Circ J 2009; 73: 662-6.

13. Daida H, Miyauchi K, Ogawa H, et al. Management and twoyear long-term clinical outcome of acute coronary syndrome in Japan. Circ J 2013; 77: 934-43.

14. Thygesen K, Alpert JS, Jaffe AS, et al. Fourth universal definition of myocardial infarction (2018). J Am Coll Cardiol 2018; 72: 2231-64.

15. Chaudhry SI, Khan RF, Chen J, et al. National trends in recurrent AMI hospitalizations 1 year after acute myocardial infarction in Medicare beneficiaries: 1999-2010. J Am Heart Assoc 2014; 3: e001197.

16. Buch P, Rasmussen S, Gislason GH, et al. Temporal decline in the prognostic impact of a recurrent acute myocardial infarction 1985 to 2002 . Heart 2007; 93: 210-5.

17. Thune JJ, Signorovitch JE, Kober L, et al. Predictors and prognostic impact of recurrent myocardial infarction in patients with left ventricular dysfunction, heart failure, or both following a first myocardial infarction. Eur J Heart Fail 2011; 13: 148-53.

18. Nakatani D, Sakata Y, Suna S, et al. Incidence, predictors, and subsequent mortality risk of recurrent myocardial infarction in patients following discharge for acute myocardial infarction. Circ J 2013; 77: 439-46. 